\title{
Alternativas para modelos de gestión del conocimiento en Instituciones de Educación Superior
}

\author{
Alternatives for knowledge management models in \\ Higher Education Institutions \\ Yesenia Acevedo-Correa $^{1 *} \quad$ Alejandro Valencia-Arias ${ }^{2} \quad$ Lemy Bran-Piedrahita $^{3}$ \\ Sergio Gómez-Molina ${ }^{3} \quad$ Claudia Arias-Arciniegas ${ }^{3}$ \\ Recibido 19 de abril de 2018, aceptado 20 de agosto de 2018 \\ Received: April 19, 2018 Accepted: August 20, 2018
}

\begin{abstract}
RESUMEN
El conocimiento concebido dentro de las Instituciones de Educación Superior debe ser compartido y apropiado por las diferentes comunidades, por lo que es un reto identificar mecanismos para gestionarlo como un activo intangible generador de ventajas competitivas. Dada esta necesidad, este artículo tiene como objetivo identificar diferentes estructuras de modelos de gestión del conocimiento en Instituciones de Educación Superior, con el fin de brindar los principales criterios a tener en cuenta al momento de implementar sistemas de gestión del conocimiento en instituciones universitarias y comprender la evolución de esta temática investigativa. La metodología se desarrolló a través de una revisión sistemática de artículos científicos, con la aplicación de una ecuación de búsqueda en la base de datos Scopus. En total se hizo un análisis realizado a 22 artículos, donde se identificaron los tipos de modelos de gestión del conocimiento utilizados, sus objetivos, las variables implicadas, la descripción del modelo, los instrumentos metodológicos referenciados en su elaboración, su evolución y sus aspectos diferenciadores. De esta forma la presente investigación pretende aportar una guía académica y práctica para Instituciones de Educación Superior que busquen adaptar, implementar o crear modelos de gestión del conocimiento. Se concluye que la evolución de los modelos se ha orientado hacia la incorporación de nuevas plataformas tecnológicas, nuevas técnicas y una búsqueda por articular de mejor forma los actores involucrados en el proceso de gestión del conocimiento.
\end{abstract}

Palabras clave: Sistemas de gestión del conocimiento, universidad, conocimiento tácito, conocimiento explícito, revisión sistemática.

\section{ABSTRACT}

The knowledge produced within Higher Education Institutions must be shared and appropriate by the different communities, so it is a challenge to identify mechanisms to manage this type of knowledge as an intangible asset generating competitive advantages. Given this need, this article aims to identify different

1 Fundación Universitaria Católica del Norte. Coordinación General de Investigaciones. Medellín, Colombia. E-mail: yacevedoc@ucn.edu.co

2 Instituto Tecnológico Metropolitano. Departamento en Ciencias Administrativas 3 Dirección: Cl. 54a \#30-99. Medellín, Colombia. E-mail: jhoanyvalencia@itm.edu.co

3 Fundación Universitaria Católica del Norte. Dirección: Calle 52 № 47-42 Edificio Coltejer Piso 5. Medellín, Colombia. E-mail: lbpiedrahita@ucn.edu.co; sgmolina@ucn.edu.co; cmariasa@ucn.edu.co

* Autor de correspondencia: yacevedoc@ucn.edu.co 
structures of knowledge management models in Higher Education Institutions, in order to provide the main criteria to be taken into account when implementing knowledge management systems in university institutions and understand the evolution of this research topic. The methodology was developed through a systematic review of scientific articles, with the application of a search equation in the Scopus database. A total of 22 articles were analysed, identifying the types of knowledge management models used, their objectives, the variables involved, the description of the model, the methodological tools involved in its elaboration, its evolution and its differentiating aspects. In this way, this research seeks to be an academic and practical guide for Higher Education Institutions seeking to adapt, implement or create models of knowledge management. It is concluded that the evolution of the models has been oriented towards the incorporation of new technological platforms, new techniques and a search to better articulate the actors involved in the knowledge management process.

Keywords: Knowledge management systems, higher education institutions, tacit knowledge, explicit knowledge, systematic review.

\section{INTRODUCCIÓN}

La gestión del conocimiento tiene el propósito de recolectar, organizar, distribuir, compartir y emplear los bienes intangibles de una organización, tales como recursos de conocimiento y relaciones, estos deben beneficiar a sus miembros. En ese sentido, una actividad que se centra en el conocimiento implica un intercambio de este dentro de la institución y en ese proceso, se contribuye a su aplicación e innovación [1].

Las Instituciones de Educación superior (IES), son espacios de generación de conocimiento, puesto que son organizaciones dotadas de fuerza creativa. Sin embargo, la gestión de este es complicada, ya que es difícil de almacenar en repositorios, porque el conocimiento se usa sólo como una respuesta a necesidades específicas, por consiguiente, se resuelven inconvenientes según surjan en el día a día y no se brinda información del conocimiento que debe ser transferido o reutilizado, lo que requiere grandes esfuerzos en realización de búsquedas. Hay entonces, una baja eficiencia de los motores de búsqueda asociada a los repositorios, debido a que proporciona información a sujetos específicos, lo que lleva a que tome más tiempo en identificar lo que necesita [1].

De esa manera, es necesario que el conocimiento producido dentro de las IES, sea compartido y apropiado, por lo que este debe ser administrado adecuadamente, teniendo en cuenta que se constituye como un activo intangible generador de ventajas competitivas [2]. Para ello, la gestión del conocimiento (GC) se basa en la estructura de la organización, vista como un sistema, con la intención de identificar elementos de conocimiento para establecer mecanismos que lo explique y faciliten su acceso [2]. Más aún, cuando se quiere estar en sintonía con el mundo globalizado y pertenecer a la élite de clase mundial en el que se encuentran inmersas estas organizaciones, se debe potenciar su capacidad investigativa, volviéndose una prioridad institucional. Por lo tanto, las IES en su interacción con la comunidad, crean nuevo conocimiento y nuevas tecnologías que brindan un almacén de conocimiento y de capacidades que la sociedad pueda aprovechar, enfatizando en la relación educación-conocimiento [3].

Así, la GC, se comprende como el más alto nivel organizativo, pues antes de llegar a este estadio, las instituciones buscan resolver inconvenientes en razón a certificaciones para brindar servicios de calidad, integrando sistemas de información, para fortalecer la conectividad, creando bases de datos y proporcionando información para ser compartida, garantizando el acceso y actualización permanente de bancos de almacenamiento como la Intranet [4-5].

En el contexto universitario, se plantean modelos de gestión del conocimiento en la búsqueda por promover el aprendizaje organizacional, aprovechando las habilidades específicas de quienes pertenecen a este (estudiantes, docentes, personal administrativo), con la intención de que el conocimiento individual sea un saber institucional, lo que se da a través de asumir la cultura que refuerce su relación con otros grupos con los que se interactúe interna y 
externamente [4, 6-7]. Por ende, se han planteado diversos sistemas de gestión de conocimiento [2, 8-9, 12] para el contexto de las IES que, en general, tienen la intención de generar estrategias que lleven, tanto a estudiantes como a personal administrativo a concientizarse del conocimiento que producen y que debe ser transferido en estas instituciones.

Entre los que más se destacan, se encuentra el de Nonaka y Takeuchi [8] que se refiere a que el conocimiento se crea a partir de un movimiento cíclico en espiral, en el cual interactúan conocimientos tácitos (empíricos) y explícitos (formalizados) en las dimensiones de la organización; aunque este no se formula directamente para el contexto educativo, aparece como una referencia constante en trabajos posteriores a este, sirviendo como punto de partida para proponer otros, que luego se enfocan en vincular herramientas tecnológicas para permitir la circulación del conocimiento en las instituciones, utilizando minería de datos y diferentes estrategias para la GC, entre esos se encuentran los de Natek y Zwilling [12] y Alammari y Chandran [13]. También se plantean sistemas de gestión del conocimiento con el fin de identificar el conocimiento en razón al desempeño de los procesos en la estructura organizacional, a través de un análisis de contexto interno y externo, con la ayuda de minería de datos y creación e implementación de software que sustenten los sistemas de gestión de conocimiento presentados $[2,9,14]$.

A partir de lo anterior, se observa que son cada vez más las herramientas y opciones que tienen las IES para una adecuada gestión del conocimiento y que los modelos que se han desarrollado o ajustado frente al tema responden a necesidades que pueden ser comunes a diferentes contextos de la educación superior. Dado lo anterior, se hace necesario examinar diferentes estructuras de modelos de gestión del conocimiento que se han implementado en IES y estudiar cómo ha sido la evolución de esta temática investigativa de tal forma que se pueda construir una base académica clara para la formulación o implementación de nuevos modelos de gestión del conocimiento. Con el fin de aportar a esta necesidad, se plantea una revisión sistemática de artículos publicados en revistas indexadas en Scopus donde a partir de una ecuación de búsqueda se realizó el análisis a 22 artículos, identificando los insumos primordiales para la elaboración de un adecuado modelo de gestión del conocimiento en IES y mostrando las tendencias investigativas en este campo del saber.

\section{METODOLOGIA}

Para lograr el propósito de este artículo, se plantea una revisión sistemática de artículos publicados en la base de datos Scopus, aplicando criterios para filtrar información como la definición de preguntas orientadoras, estrategias de búsqueda, para obtener la información acorde a la intención del análisis. Este se desarrolla de acuerdo a los tipos de modelos encontrados, autores utilizados y país en el que se realizan los estudios.

La revisión sistemática de literatura, trata de "resumir, compilar, criticar y sintetizar la investigación existente sobre un área temática o fenómeno de interés usando un proceso de búsqueda, catalogación, ordenamiento, análisis, crítica y síntesis; las revisiones de literatura son contribuciones al conocimiento actual ya que sus hallazgos son únicamente obtenidos cuando la literatura más relevante es analizada como un todo y no como la simple lectura de documentos aislados" [15].

Para lograr esa revisión, se aplicó una ecuación de búsqueda en la base de datos SCOPUS el 27 de julio de 2017, orientada a la gestión del conocimiento como sistema en el contexto de las instituciones de educación superior, dichos términos fueron buscados en los campos título y palabra clave, y no hubo restricción de período de tiempo para obtener un horizonte más amplio de análisis. Se ha elegido la base de datos de Scopus ya que esta proporciona herramientas para gestionar la información y cumple otros criterios como la cantidad de citaciones y la accesibilidad que la hacen ser más usada en este tipo de análisis en la literatura [31].

Consecuencia de eso, se obtuvieron 43 resultados entre artículos científicos y artículos de conferencia, de los cuáles se decidió trabajar con 22, dejando de lado 21, debido a que no cumplían con afinidad al tema de interés, pues no se interesaban por trabajarlo desde IES o porque también se encontraron libros que recogían ponencias que ya aparecían dentro del conjunto de documentos de los resultados. Por lo tanto, se definieron como criterios de inclusión que los artículos trataran un enfoque investigativo 
de GC en IES, que se desarrollara un modelo o sistema de gestión del conocimiento y que fueran artículos resultados de investigación científica. Así, la ecuación aplicada fue la siguiente:

\section{TITLE-ABS-KEY ( $\{$ KNOWLEDGE MANAGEMENT $\}$ W/1 \{SYSTEM $\})$ AND TITLE-ABS-KEY (\{Universit* $\}$ ) OR TITLE- ABS-KEY (\{HIGHER EDUCATION $\})$}

Para tratar los modelos encontrados en los artículos revisados, se utilizó una matriz de datos en Excel, en la que se clasificó información de los artículos para identificar si se proponía un modelo de GC, si se adaptaba a un contexto específico o si se planteaba uno propio, para ello se identificaron los autores que fundamentaban las propuestas encontradas, su objetivo, la población objeto del modelo, estrategias, sus aportes, país de aplicación, la agenda que surge de ellos, entre otras, lo que permitió identificar tendencias en la formulación y aplicación de estos sistemas para determinar el cómo estos han evolucionado. A partir de eso, se tabularon datos relevantes que sirvieron para el análisis y presentación de los resultados de acuerdo al interés de este escrito.

\section{RESULTADOS}

Objetivos en los que se orientan los modelos bajo análisis de Gestión de Conocimiento en las Instituciones de Educación Superior

Los objetivos que impulsaron el desarrollo de los 22 modelos bajo análisis son muy variados, sin embargo, algunos de ellos apuntan a gestionar los diferentes flujos de conocimiento que intervienen en la universidad de tal manera que generen valor. Por otro lado, hay objetivos orientados en implementar buenas prácticas en torno a la gestión del conocimiento que permitan aprender de otras acciones exitosas.

Otro objetivo en el que se orientan algunos modelos es en hacer uso de técnicas de minería de datos con el fin de analizar grandes volúmenes de datos provenientes de estudiantes y cursos, de modo que contribuyan al mejoramiento de la GC en las instituciones de educación superior. Finalmente, otro de los enfoques que presentan los objetivos es diseñar e implementar Sistemas para la Gestión del Conocimiento (KMS) que permitan hacer mejor uso del conocimiento en las IES y se adecuen a ambientes colaborativos de aprendizaje.

\section{Periodo de análisis de los modelos de Gestión de Conocimiento en las Instituciones de Educación Superior}

Fuente: Elaboración propia.

\section{Período de análisis}

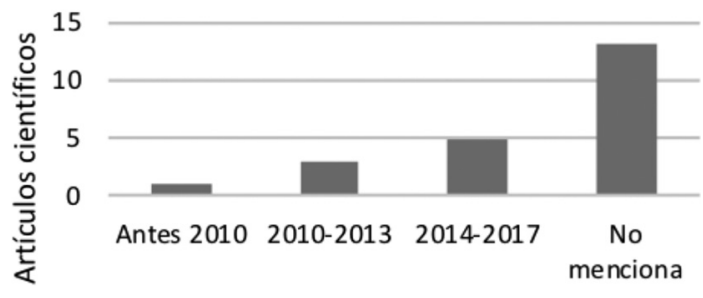

Figura 1. Período de análisis de los modelos.

En este punto se busca identificar si las propuestas de modelo surgen a partir de una recopilación de información específica en un periodo de tiempo determinado o si se plantean como una propuesta genérica (en su mayoría conceptuales) para ser implementados en otros contextos más generales. A partir de la Figura 1, se puede observar que la mayoría de artículos científicos bajo análisis no reportan recolección de información o validación en un período de tiempo específico, esto podría explicarse por el deseo de los autores de formular modelos atemporales con el fin de que tengan una mayor vigencia en el tiempo. Por otro lado, entre los artículos que, si mencionan su período de análisis, los más frecuentes son los artículos enmarcados en un período actual (2014-2017), mostrando que en los últimos años existe una mayor tendencia por realizar investigaciones alrededor de la formulación y adopción de modelos de gestión de conocimiento en Instituciones de Educación Superior. Esto muestra vigencia en la temática y da indicios de la necesidad de explorar de manera permanente nuevos ajustes y propuestas de nuevos constructos para los modelos.

Países de los cuales han surgido los modelos bajo análisis de Gestión de Conocimiento en las Instituciones de Educación Superior

En la Tabla 1 se pueden apreciar los países donde han surgido o se han desarrollado los 22 modelos bajo análisis. De esta tabla se puede observar que estos se han originado a partir de una diversidad 
de países, tanto de economías desarrolladas como economías de mercados emergentes y en desarrollo. En este sentido, se puede decir que la temática sobre modelos de gestión de conocimiento en Instituciones de Educación Superior no es ajena o no excluye cierto tipo de mercados o países, sino que ha sido de interés de una amplia gama de regiones diferentes entre sí que han investigado y desarrollado modelos en torno a esta temática, con el fin de atender y responder a las particularidades sociales, culturales y economías de cada país. La Tabla 1 ha tomado como referencia el Índice de Desarrollo Humano (IDH) del banco mundial para la clasificación de los países.

Tabla 1. Países donde se han desarrollado los modelos.

\begin{tabular}{|l|l|l|}
\hline Altos ingresos & $\begin{array}{c}\text { Medio-altos } \\
\text { ingresos }\end{array}$ & $\begin{array}{c}\text { Medio-bajos } \\
\text { ingresos }\end{array}$ \\
\hline España & China & Indonesia \\
\hline Estados Unidos & Colombia & India \\
\hline Eslovenia & Tailandia & \\
\hline Taiwán & Rusia & \\
\hline Arabia Saudita & Malasia & \\
\hline & Argelia & \\
\hline & Irán & \\
\hline
\end{tabular}

Fuente: Elaboración propia.

\section{Enfoque de los modelos bajo análisis de Gestión de Conocimiento en las Instituciones de Educación Superior}

En la Tabla 2 se pueden observar los distintos enfoques a través de los cuales se han desarrollado los 22 modelos bajo análisis. El enfoque más utilizado entre estos modelos es el cuantitativo, el cuál a diferencia del enfoque cualitativo, proporciona un alto nivel de precisión de medición y poder estadístico [16], además se dice que mediante este enfoque se puede llegar a conclusiones amplias y generales rápidamente [17], dado que es posible recibir las respuestas de una gran magnitud de personas a partir de una cantidad limitada de preguntas [18]. Contrario al enfoque cuantitativo, los métodos de investigación cualitativa proporcionan una gran cantidad de datos detallados sobre un número mucho menor de personas [17], sin embargo, como se puede observar en la Tabla 3, este es el enfoque menos utilizado en los artículos sobre modelos de gestión del conocimiento.
Tabla 2. Tipo de enfoques utilizados en los modelos.

\begin{tabular}{|l|c|}
\hline Tipo de enfoque & Total artículos \\
\hline Mixto & 7 \\
\hline Cualitativo & 2 \\
\hline Cuantitativo & 10 \\
\hline No menciona & 3 \\
\hline
\end{tabular}

Fuente: Elaboración propia.

En la Tabla 2 también se observa que el enfoque mixto es bastante utilizado en los modelos de gestión de conocimiento en IES, dado que se ha señalado que una de las ventajas este enfoque es la "facilidad para generar y verificar teorías en un mismo estudio, la posibilidad de obtener inferencias más fuertes" [19]. De esta manera, la combinación e integración del enfoque cuantitativo y cualitativo enriquece la investigación haciéndola más amplia y completa. A través del enfoque mixto se generan más dimensiones para comprender el fenómeno en cuestión a partir de un espectro más amplio de preguntas que permiten dar respuestas más contundentes al problema bajo estudio [20].

En este sentido, ha surgido la necesidad de que los modelos de gestión del conocimiento en IES surjan desde enfoques mixtos con el fin de tener una más amplia perspectiva de todos los factores que influyen en estos, pues no basta simplemente con encontrar el problema, sino en ir más allá y entender su razón de ser. Por esto, se ha señalado que resulta útil emplear un enfoque estructurado (cuantitativo) para revelar problemas, y cuando ya éste se haya identificado, es conveniente utilizar un método no estructurado (cualitativo) con el fin de explorar la naturaleza del problema [21].

Instrumentos metodológicos de los modelos bajo análisis de Gestión de Conocimiento en las Instituciones de Educación Superior

En la Tabla 3 se pueden ver los diferentes instrumentos metodológicos que se han utilizado en los artículos analizados, con el fin de construir y desarrollar los modelos de gestión de conocimiento en las IES. Se puede observar que la aplicación de encuestas es la metodología más frecuente, las cuales son instrumentos que se aplican con el fin de descubrir tendencias generales a partir de las opiniones, experiencias y comportamiento de las personas, por lo que se dice que son útiles para aprender patrones 
Tabla 3. Instrumentos metodológicos utilizados en los modelos.

\begin{tabular}{|l|c|}
\hline \multicolumn{1}{|c|}{ Tipo de Metodología } & $\begin{array}{c}\text { Total } \\
\text { artículos }\end{array}$ \\
\hline Encuestas & 8 \\
\hline Encuestas y entrevistas & 3 \\
\hline Encuestas y repositorio & 1 \\
\hline Entrevistas & 1 \\
\hline Entrevistas y grupos focales & 1 \\
\hline Grupos focales y observación & 1 \\
\hline Fuentes secundarias & 1 \\
\hline Fuentes secundarias y repositorios virtuales & 1 \\
\hline No mencionados & 5 \\
\hline
\end{tabular}

Fuente: Elaboración propia.

generales tomando como muestra una cantidad considerable de individuos [22]. En contraste, las entrevistas resultan útiles para obtener detalles de unas cuantas personas o cuando se desea saber exactamente la opinión de expertos sobre algún tema [22], sin embargo en el caso de modelos de gestión de conocimientos en IES no es muy común su aplicación, pero si es frecuente que las entrevistas sean complementarias de las encuestas con el fin de definir una metodología a partir de dos instrumentos que permitan obtener información de una gran cantidad de personas que tienen vínculo con las IES pero a la vez se logre tener información detallada para la construcción del modelo, la cual la provee en gran medida las entrevistas.

En la Tabla 3 también se puede observar otro tipo de instrumentos metodológicos que han sido utilizados, aunque en menor medida en comparación con las encuestas y entrevistas que son las más frecuentes. Entre estos, se encuentran los grupos focales, de los cuales se ha señalado que permiten "obtener datos con un nivel de profundidad al que no se puede acceder desde otras técnicas o metodologías" [23]. También se ha hecho uso de fuentes secundarias y repositorios como métodos para la recolección de información.

\section{Relación de modelos utilizados para la elaboración de modelos de gestión del conocimiento en Instituciones de Educación Superior}

En la Figura 2, se observan tres filas alineadas jerárquicamente, relacionadas a los objetivos que se identifican en modelos de GC. En la primera
Fuente: Elaboración propia.

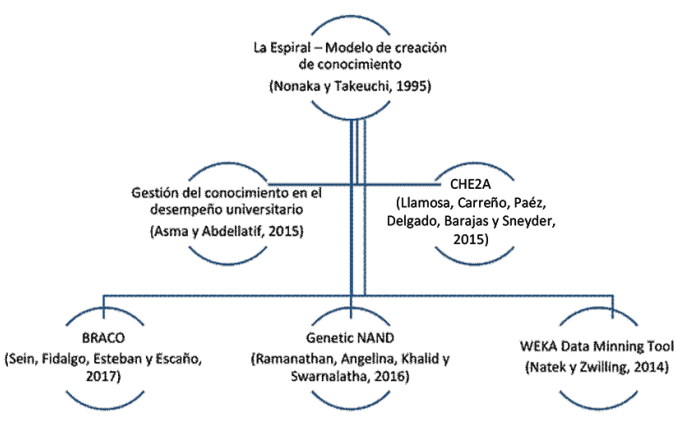

Figura 2. Relación de modelos encontrados.

se encuentra el modelo de Nonaka y Takeuchi [8], como modelo seminal y que marca el cómo se llegan a generar otros sistemas de gestión del conocimiento. Estos autores plantean que el conocimiento es tácito (empírico, experiencias) o explícito (expresado formalmente) y este se desenvuelve en dos dimensiones, una de ellas es la ontológica que se refiere a los niveles en los que se genera conocimiento (individual, grupal, organizacional e interorganizacional) y la otra es la epistemológica en la que interactúan los dos tipos de conocimiento (socialización, exteriorización, combinación e interiorización) [8].

En la segunda fila, se tienen dos ejemplos de modelos que se basan en el de Nonaka y Takeuchi, pero lo modifican para emplearlo según el objetivo con el que quiere implementar un sistema de gestión del conocimiento en el contexto universitario. En este caso, se emplean diferentes etapas para mejorar la experiencia de gestionar el conocimiento dentro de las IES, basándose en la interacción entre conocimientos tácitos y explícitos de acuerdo a las dimensiones ontológicas y epistemológicas para la creación de estos $[2,9]$.

Se analiza el contexto externo para tomar decisiones respecto al cómo desarrollar habilidades de GC en la universidad. El entorno en el que se desenvuelven los estudiantes, profesores y administrativos es relevante para identificar cómo gestionar conocimiento, así se reconoce la experiencia organizacional y con el apoyo de la innovación que surge en las IES, se pueden resolver problemas [9], utilizando modelos ontológicos y epistemológicos para comprender los ciclos que se desarrollan en las universidades. En ese sentido, los líderes son quienes se encargan de 
los recursos disponibles para la implementación de KMS y predecir estrategias y conocer las necesidades de los actores implicados [11].

En el tercer nivel, se ubican algunos modelos que se interesan por desarrollar ventajas en las IES a partir de la utilización de minería de datos que es una estrategia que trata de extraer el conocimiento que se condensa en bases de datos para la toma de decisiones. Esta no se debe confundir con un gran software, sino que usa diferentes aplicaciones por las áreas que participan de las etapas de análisis [24]. Por lo tanto, se hace gestión del conocimiento utilizando minería de pequeños grupos de datos para identificar la información y posteriormente el conocimiento sustancial de la institución [12].

\section{Descripción de los modelos presentados}

En la Tabla 4 se presenta la descripción de algunos de los modelos identificados en la revisión sistemática, con el fin de soportar lo ya presentado en el segmento anterior.

Ahora bien, según la Tabla 4, la descripción de los modelos presentados permite determinar que se han gestado algunos lineamientos que serían primordiales al crear sistemas de gestión del conocimiento, puesto que primero se debe identificar el contexto en el que se aplicarán, se seleccionan los actores que participarán y de acuerdo a eso, se llevan a cabo actividades de recolección de información para reconocer cómo se encuentra el conocimiento dentro de la organización con el objetivo de generarle mayor valor a la organización por medio de apalancar sus competencias en el mercado, esto se ha realizado con la aplicación de estrategias de GC como comunidades de práctica, recuperación de buenas prácticas, repositorios electrónicos y minería de datos. En el caso de las IES, se da una preocupación explícita frente al desempeño estudiantil y docente, alineado con las funciones sustantivas de las universidades (docencia, investigación y extensión).

\section{Estrategias identificadas en los modelos encontrados según enfoque de los modelos revisados}

En la Tabla 5, se observa la relación existente entre enfoques de investigación y las estrategias utilizadas para promover la GC en las IES. Para cualquier caso, se emplean diversas técnicas de recolección
Tabla 4. Descripción de modelos.

\begin{tabular}{|c|c|}
\hline Modelo & Descripción del modelo \\
\hline Espiral [8] & $\begin{array}{l}\text { Propone la interacción de conocimientos } \\
\text { tácitos y explícitos en dos dimensiones } \\
\text { (ontológica y epistemológica) como } \\
\text { proceso para la generación de conocimiento } \\
\text { para darle valor a las organizaciones. }\end{array}$ \\
\hline $\begin{array}{l}\text { Gestión del } \\
\text { conocimiento en } \\
\text { el desempeño } \\
\text { universitario [2] }\end{array}$ & $\begin{array}{l}\text { Se propone un modelo para el mejoramiento } \\
\text { sistemático. Se incluyen: factores de GC } \\
\text { (entradas), funciones de GC (procesos) y } \\
\text { desempeño organizacional (PR) (salidas). }\end{array}$ \\
\hline $\mathrm{CHE}$ & $\begin{array}{l}\text { CHE2A es un KMS enriquecido con la } \\
\text { experiencia de las organizaciones y apoya } \\
\text { la innovación en educación superior a } \\
\text { través del desempeño del servicio. Este } \\
\text { promueve una nueva manera de pensar } \\
\text { para resolver grandes problemas en la } \\
\text { interacción con la tecnología. }\end{array}$ \\
\hline BRACO [10] & $\begin{array}{l}\text { Identificar los principios de gestión del } \\
\text { conocimiento para la producción de } \\
\text { estas instituciones. Se basa en el modelo } \\
\text { de Lewin, adaptado por Fidalgo et. Al, } \\
\text { agregando dos etapas de mejora desde } \\
\text { la experiencia vivida. El KMS llamado } \\
\text { Braco, lleva a que fluya el conocimiento } \\
\text { entre las diferentes etapas de investigación } \\
\text { para la acción. }\end{array}$ \\
\hline $\begin{array}{l}\text { WEKA Data } \\
\text { Minning } \\
\text { Tool [12] }\end{array}$ & $\begin{array}{l}\text { El modelo de predicción de Data Mining } \\
\text { Fill es un modelo predictivo para visualizar } \\
\text { un "futuro estudiante de grado final", } \\
\text { basado en instancias ya conocidas. } \\
\text { La primera suposición mostró que los } \\
\text { resultados pasados fueron considerados } \\
\text { como buenos predictores del futuro. }\end{array}$ \\
\hline
\end{tabular}

Fuente: Elaboración propia.

Tabla 5. Estrategias aplicadas en modelos identificados según el enfoque metodológico.

\begin{tabular}{|l|l|}
\hline $\begin{array}{c}\text { Enfoque de } \\
\text { los modelos }\end{array}$ & \multicolumn{1}{c|}{ Estrategias aplicadas } \\
\hline Cuantitativo & $\begin{array}{l}\text { Comunidades de práctica. Minería de datos. } \\
\text { Repositorios electrónicos Tutorías. }\end{array}$ \\
\hline Cualitativo & $\begin{array}{l}\text { Mapas de conocimiento. } \\
\text { Comunidades de práctica. }\end{array}$ \\
\hline Mixto & $\begin{array}{l}\text { Recuperación de buenas prácticas. } \\
\text { Tutorías. }\end{array}$ \\
\hline
\end{tabular}

Fuente: Elaboración propia.

de información para reconocer el contexto en el que se aplicará el KMS con intención de reconocer donde intervenir para mejorar el desempeño de las IES y de allí determinar las mejores estrategias para 
implementar sistemas de gestión del conocimiento en este contexto.

Se tiene entonces, que las estrategias confluyen entre sí, dado que estas se han utilizado para fomentar la GC en diferentes campos, todas están adaptadas al entorno de interés, puesto que lo que más varía es la manera en la que estas se aplican, mediados por las innovaciones tecnológicas y los actores que participan del modelo.

\section{Evolución en los modelos revisados}

En la Figura 3 se observa que la fortaleza de los modelos de gestión del conocimiento, en un inicio, se orientaba hacia su fundamentación conceptual, destacando las etapas en las que se crea el conocimiento organizacional de interés en los diferentes grupos que pertenecen a una institución $[10,8]$. Seguidamente, las plataformas tecnológicas que permiten el almacenamiento de conocimiento se gestaron como foco principal en estos modelos, de manera que estas sirvieran como un medio para potenciar la transferencia del conocimiento organizacional [2], en los que también se aplicaban estudios para conocer el entorno en el que se encuentran las instituciones, lo que permite determinar el cómo interactúa una institución con el exterior. En esta época, se comienza a explorar la minería de datos para aplicarla como un proceso que contribuye a depurar la información almacenada virtualmente para determinar el conocimiento relevante producido organizacionalmente [12]. Luego, hay un interés por hacer medibles los sistemas de gestión del conocimiento, por medio del fortalecimiento de la interacción y de la confianza que se gesta a partir de las estrategias implementadas [25] presentando modelos más complejos basados en software y minería de datos para así visibilizar con mayor precisión el impacto que estos tienen $[2,12]$.

Todo lo anterior, conlleva a pensar a que se le da importancia a la gestión tecnológica como primer paso para la consolidación de sistemas de gestión del conocimiento, puesto que esta se constituye en la base para su apropiación dentro de las IES, sin dejar de lado la validez que estos obtienen con las relaciones sociales que se gestan alrededor de los modelos, necesarias para potenciar la circulación del conocimiento.

\section{Aspectos diferenciadores}

El ejercicio llevado a cabo con esta revisión sistemática de literatura, posibilita proveer al lector un encuentro entre las diferentes corrientes que

Fuente: Elaboración propia.

\section{5-1995}

Los modelos de gestión del conocimiento se basan en su aspecto conceptual y este les genera el rigor necesario para aplicar GC en IES. Se trata de la comprensión de los términos y de aplicaciones de acuerdo a lo teórico

\section{6-2005}

Se genera una preocupación por gestionar el conocimiento de la mano con repositorios tecnológicos y plataformas que permitan el flujo de información, utilizando software tecnológicos para la transferencia y apropiación del conocimiento. Se analiza el entorno externo en el que se ubica la institución.

\section{6-2016}

Se aplica la minería de datos como estrategia para gestionar el conocimiento en IES, con el fin de depurar datos y obtener el conocimiento más relevante en las instituciones para generarle mayores ventajas competitivas y así generar mayores niveles de medición y cuantificación de la GC.

Figura 3. Evolución de sistemas de gestión de conocimiento según los modelos revisados. 
han abordado la gestión del conocimiento desde la perspectiva de las Instituciones de Educación Superior; develando así ser un campo de interés en todas las latitudes, sin que exista injerencia del contexto social, cultural o económico de donde vengan las contribuciones; lo cual pone en manifiesto el interés global que existe por esta temática, máxime en una sociedad cada vez más globalizada e interconectada, donde las Tecnologías de la Información y la Comunicación -TIC- han jugado un papel clave, con lo que más allá de exponerse el capital existente en esta área del conocimiento, se vislumbran retos y oportunidades para la comunidad académica, especialmente en la capacidad de contribuir al encadenamiento con el sector productivo, y así dinamizar las economías.

De este modo, entre los modelos revisados, se encuentran como aspectos diferenciadores la investigación para la acción transformadora en el sentido de adaptación de la espiral que proponen $[1,8,26]$, como primera instancia para formular sistemas de gestión del conocimiento, pues estos autores con el desarrollo de los tipos de conocimiento, marcaron una línea de trabajo que se observa en los resultados arrojados por la ecuación creada para este estudio; fijando así las bases que han servido para el desarrollo de nuevas aproximaciones al campo de conocimiento.

Así mismo, la generación de KMS en relación a los currículos universitarios es otro factor diferenciador relevante, pues de esa manera se brindan canales de transferencia de conocimiento no sólo interna sino también externamente [27], así como encontrar las maneras de predecir comportamientos de estudiantes frente a su desempeño [11-12, 28]. En adición, es posible visibilizar las barreras que se interponen para el intercambio de conocimiento y que dificultan los lazos de confianza y la interacción, así como internalizar procesos de acuerdo a los objetivos de las IES [3, 9].

Por otra parte, uno de los elementos que se mantiene en muchos de estos modelos, es el uso de la virtualidad como plataforma en la que se comparten los conocimientos, por medio de comunidades virtuales, tutorías, acompañamiento y supervisión para el almacenamiento de los datos respectivos a la GC y que contribuyen a mejorar los servicios ofrecidos por las IES; con lo cual se hace evidente el rol que ejercen en la actualidad las TIC, además de proveer una ruta de incorporación de estas tecnologías en el momento de planear, diseñar y ejecutar modelos de gestión del conocimiento al interior de las IES.

Adicionalmente, el estudio realizado devela no sólo la necesidad de incorporar las nuevas tecnologías a los procesos afines a la GC, sino que también provee como ya se ha expuesto los principales autores que sirven como referente bien sea para la adaptación de modelos a los contextos en que se quieran llevar, e incluso, las principales herramientas utilizadas para la construcción de éstos, que como lo arroja la literatura consultada son preferentemente la encuesta y en algunos casos, las entrevistas como técnica auxiliar para profundizar en el ejercicio de diseño de KMS.

Por tanto, puede afirmarse que lo expuesto a lo largo de este manuscrito, se constituye en un insumo que sirve como base de discusión sobre la temática, mostrando las contribuciones más significativas que se han hecho en el campo de la gestión del conocimiento, sirviendo a su vez como plataforma para hacer más visibles las aproximaciones existentes; e igualmente, como referente para la toma de decisiones en académicos e Instituciones de Educación Superior. Para los primeros, permite ver los retos existentes -particularmente para la región latinoamericana, que, de acuerdo al ejercicio realizado, no presenta contribuciones marcadas en este campo-, frente a las formas en que se ha abordado la temática e insumos para llevar a estos a la creación de nuevas propuestas, sin desconocer el camino recorrido por otros estudiosos. Y para las segundas, suministra información clave para la toma de decisiones, al condensar los principales referentes teóricos, evolución de la temática en el tiempo, así como los enfoques y técnicas de recolección de información empleados; con lo que es posible tener mayor certeza en el proceso de diseño y planeación de futuros modelos de gestión del conocimiento para el contexto de las IES.

\section{CONCLUSIONES}

Los diversos modelos de gestión del conocimiento identificados, se han enmarcado en la propuesta guiada por Nonaka y Takeuchi, como pilar para orientar la GC en IES, de esa forma, se mantienen intereses 
por el movimiento que se da entre conocimientos tácitos y explícitos en distintos grupos internos y externos a la organización. Además se considera, que se ha tenido una evolución en la manera en que se fundamentan dichos modelos, los cuales no han dejado de lado un hacer relacionado a lo conceptual, pasando por lo tecnológico, luego por lo medible, hasta llegar a la preocupación por incrementar los lazos de confianza entre los miembros involucrados, de esta manera, surge el componente humano que reconforta y permite que el conocimiento circule en la organización eficazmente.

En el futuro, según los modelos de gestión del conocimiento revisados, se espera que se implementen mediciones que indiquen el rendimiento que estos generan dentro de las IES, y así tener un control de lo que se debe ajustar para fortalecer los KMS, en ese sentido, se plantean modelos que implementen algoritmos de aprendizaje automático más eficientes para predecir el comportamiento académico estudiantil [11, 29]. Se espera, además, que se generen interfaces virtuales que unifiquen el flujo de información $[9,30]$. Teniendo en cuenta lo anterior, uno de los aspectos de mayor impacto será el uso de la minería de datos, y el apoyo que brindarán los repositorios educativos virtuales que se relacionen con reforzar la interacción entre los diferentes actores que tienen el papel de mentores con estudiantes, para afianzar lazos de confianza que influyen en la transferencia y apropiación de conocimiento organizacional.

Finalmente, las revisiones sistemáticas brindan orientaciones a la hora de visibilizar cómo ha evolucionado un tema de interés, el de este caso, de modelos de gestión del conocimiento en IES, pues de esa manera se pueden tomar decisiones para investigaciones futuras y determinar cómo implementar KMS en contextos similares, retomando fortalezas y hallazgos relevantes. En vista de eso, se sugiere que este tipo de estudios se continúen realizando por su valor académico según el panorama que se brinda respecto a asuntos específicos y así continuar orientando en aspectos que tal vez no se han desarrollado lo suficiente.

\section{REFERENCIAS}

[1] Á. Fidalgo-Blanco, M. Sein-Echaluce and F. García-Peñalvo. "Knowledge Spirals in
Higher Education Teaching Innovation". International Journal of Knowledge Management. Vol. $10 \mathrm{~N}^{\circ}$ 4, pp. 16-37. 2014.

[2] K. Asma and M. Abdellatif. "A New Model for the Impact of Knowledge Management on University Performance". Journal of Information \& Knowledge Management. Vol. $15 \mathrm{~N}^{\circ}$ 4, pp. 1650041. 2016.

[3] P. Upadhyaya and R. Pillai. "Research knowledge management system in university: Faculty intention and potential barriers". IJABER. Vol. $14 \mathrm{~N}^{\circ} 12.2016$.

[4] A. Rodríguez, A. Araujo y J. Urrutia. "La gestión del conocimiento científicotécnico en la Universidad: un caso y un proyecto" Cuadernos de Gestión. Vol. 1 No 10, pp. 13-10. 2001.

[5] A.L. González, J.P. Castro y M. Roncallo. "Diagnóstico de la gestión del conocimiento en una empresa grande de Barranquilla (Colombia)" Ingeniería y Desarrollo. Vol. 16, pp. 70-103. Universidad del Norte. 2004.

[6] M. Londoño y R. Villareal. "Aproximación a un modelo de gestión de conocimiento. El caso de la Facultad de Comunicaciones de la Universidad de Antioquia". Tesis de maestría. Universidad de Antioquia. 2013.

[7] C. Valecillos y N. Quintero. "Modelo de gestión basado en el enfoque de las organizaciones y las demandas del currículo integral caso: Facultad de Ciencias Económicas y Sociales de Luz", Nigotium. Vol. 4 No 12, pp. 63-85. 2009.

[8] I. Nonaka y H. Takeuchi. "La organización creadora de conocimiento. Cómo las compañías japonesas crean la dinámica de la innovación". Oxford University Press México S.A. ISBN 970-613-454-9. 1995.

[9] R. Llamosa-Villalba, L. Carreno, Q. Paez, Q. Delgado, A. Barajas and E. Sneyder. "Enterprise architecture of Colombian Higher Education". 2015 IEEE Frontiers in Education Conference (FIE). 2015.

[10] M.L. Sein, A. Fidalgo, J. Esteban-Escaño and F. García. "The learning improvement of engineering students using peer-created complementary resources". International Journal of Engineering Education. Vol. 33 $\mathrm{N}^{\mathrm{o}}$ 2, pp. 927-937. 2017.

[11] L. Ramanathan, A. Geetha, L. Khalid and P. Swarnalatha. "A novel genetic nand paft model for enhancing the student grade performance 
system in higher educational institutions". IIOABJ. Vol. $7 \mathrm{~N}^{\circ}$ 5, pp. 1-11. 2016.

[12] S. Natek and M.Zwilling. "Student data mining solution-knowledge management system related to higher education institutions". Expert Systems with Applications. Vol. 41 No 14, pp. 6400-6407. 2014.

[13] A. Alammari and D. Chandran. "Populating contents of the Saudi eLearning objects repository 'Maknaz' from information technology \& knowledge management perspective". 2014 Asia-Pacific Conference on Computer Aided System Engineering (APCASE). 2014.

[14] O. Lezina and A. Akhterov. "Designing of the information component of pedagogical knowledge management system in a chair of technical university". 2013 International Conference on Interactive Collaborative Learning (ICL). 2013.

[15] J.D. Velásquez. "Una Guía Corta para Escribir Revisiones Sistemáticas de Literatura". Revista DYNA. Vol. $81 \mathrm{~N}^{\circ}$ 187, pp. 9-10. 2014.

[16] A.V. Matveev. "The advantages of employing quantitative and qualitative methods in intercultural research: Practical implications from the study of the perceptions of intercultural communication competence by American and Russian managers". Theory of communication and applied communication. Vol. $1 \mathrm{~N}^{\mathrm{o}}$ 6, pp. 59-67. 2002.

[17] R. Rynkun. "Collaboration between university research and industry: innovation process". Tesis de Maestría. University of Halmstad: Suecia. 2007.

[18] B. Johansson. "Quantitative or qualitative research-or a combination?”. Göteborg University: Mölndal. 1995.

[19] N. Ugalde y F. Balbastre. "Investigación Cuantitativa e Investigación Cualitativa: Buscando las Ventajas de las Diferentes Metodologías de Investigación”. Ciencias Económicas. Vol. 3 No 12, pp. 179-187. 2013.

[20] C. Miranda, J.M. Medina, L.E. Alvarado, J. Gysling, R. Rivera y A. López. "Liderazgo en docentes beneficiarios del programa de postítulos en matemáticas: un estudio evaluativo de la formación permanente de profesores". Estudios pedagógicos (Valdivia). Vol. $42 \mathrm{~N}^{\circ}$ 4, pp. 107-126. 2016.

[21] K. Eason. "Information technology and Organisational Change". Taylor \& Francis. 1988.
[22] D.L. Driscoll. "Introduction to primary research: Observations, surveys, and interviews". Writing Spaces: Readings on Writing. Vol. 2, pp. 153-174. 2012.

[23] J. Escobar y F.I. Bonilla. "Grupos focales: una guía conceptual y metodológica”. Cuadernos hispanoamericanos de psicología. Vol. 9 $\mathrm{N}^{\mathrm{o}}$ 1, pp. 51-67. 2009.

[24] Universidad Nacional del Nordeste. (2016). Minería de datos. URL: http://exa.unne.edu. ar/informatica/SO/Mineria_Datos_Vallejos. pdf

[25] B. Talebi, R. Rezayi, K. Gholizadeh, B. Heidarian and S. Zanjani. "Ranking effective factors on knowledge management system in Bonab Islamic Azad University". Life Science Journal. Vol. $9 \mathrm{~N}^{\circ}$ 4, pp. 3652-3658. 2012.

[26] P. Sriwichai, K. Meksamoot, N. Chakpitak, K. Dahal and A. Jengjalean. "The Effectiveness of "Knowledge Management System" in Research Mentoring Using Knowledge Engineering". International Education Studies. Vol. 7 N 5. 2014.

[27] Y. Tjong, H. Warnars and S. Adi. "Designing Knowledge Management model for curriculum development process: A case study in Bina Nusantara University". 2016 International Conference on Information Management and Technology (ICIMTech). 2016

[28] C. Liu, C. Lee, M. Wang, Tseng and Yi-Ling Kuo. "FML-based knowledge management system for university governance and management assessment”. 2012 IEEE International Conference on Fuzzy Systems. 2012.

[29] J. Peng, D. Jiang and X. Zhang. "Design and Implement a Knowledge Management System to Support Web-based Learning in Higher Education". Procedia Computer Science. Vol. 22 pp. 95-103. 2013.

[30] M. Simanjuntak, S. Manalu and R. Tobing. "Towards an institute of technology: a knowledge management system challenges in the perspective of academic and student administration bureau". Jurnal Teknologi. Vol. $78 \mathrm{~N}^{\mathrm{o}}$ 6-3. 2016.

[31] M. Hall. "Publish and perish? Bibliometric analysis, journal ranking and the assessment of research quality in tourism". Tourism Management. Vol. $32 \mathrm{~N}^{\circ}$ 1, pp. 16-27. 2011. 